\title{
Efecto del ácido zoledrónico en pacientes pediátricos con diagnóstico clínico de osteogénesis imperfecta: un estudio de cohorte
}

\author{
Effect of zoledronic acid in pediatric patients \\ with clinical diagnosis of osteogenesis imperfecta: a cohort study \\ Camilo E. Garcés-Constaín MD' Emilse Beltrán-Zúniga MD², \\ Juan M. Concha-Sandoval MD', Yoseth J. Ariza-Araújo MSc',
}

María A. Acosta-Aragón $\mathrm{PhD}^{5}$

\begin{abstract}
Introducción: la osteogénesis imperfecta es un trastorno congénito caracterizado por la fragilidad del hueso, causado por mutaciones en los genes del procolágeno tipo I. La incidencia es variable, encontrando un caso por cada 5.000 a 70.000 nacidos vivos. Debido a las limitaciones en el tratamiento se ha planteado el uso de bifosfonatos como el ácido zoledrónico. Objetivo: describir el efecto del tratamiento con ácido zoledrónico endovenoso en pacientes pediátricos con osteogénesis imperfecta atendidos en el Hospital Universitario San José (Popayán, Colombia) durante 2013 y 2014 . Materiales y métodos: se realizó un estudio observacional tipo cohorte de carácter cerrado y sin grupo de comparación. El tratamiento con ácido zoledrónico se realizó trimestralmente durante un año, tiempo en el que se determinaron los efectos positivos y adversos del tratamiento. Resultados: seis pacientes presentaron diagnóstico de osteogénesis imperfecta; uno fue excluido porque inició tratamiento quirúrgico, los demás iniciaron tratamiento con ácido zoledrónico. Un paciente se retiró voluntariamente y cuatro completaron el estudio, dos sin fracturas y dos con una fractura durante este período. La densidad mineral ósea de la columna lumbar y femoral presentó mejoría, documentándose en dos pacientes valores normales a los tres meses de finalizado el tratamiento. La intensidad del dolor óseo, según la escala visual análoga al inicio del estudio, fue entre 5 y 8 , y al final entre 0 y 2. No se encontraron cambios en la limitación funcional ni efectos adversos. Conclusiones: la terapia con ácido zoledrónico endovenoso en pacientes con osteogénesis imperfecta mejora el curso de la enfermedad independientemente de la severidad.
\end{abstract}

Palabras clave: osteogénesis imperfecta, bifosfonatos, fracturas óseas, niño.

Introduction: osteogenesis imperfecta is a congenital disorder characterized by bone fragility, caused by mutations in genes encoding the type I procollagen. The incidence is variable with ranges reported from one case per 5,000 to 70,000 live births. Due to limitations in the treatment, it has proposed the use of bisphosphonate as zoledronic acid. Objective: to describe the effect of treatment with intravenous zoledronic acid in of pediatric patients diagnosed with osteogenesis imperfecta attended at the Hospital Universitario San José (Popayán,

\footnotetext{
' Médico, residente en Pediatría, Universidad del Cauca. Popayán, Colombia. Correspondencia: Carrera 6 Número 10 N-142. Departamento de Pediatría, Hospital Universitario San José. Correo electrónico: cgarces@unicauca.com

${ }_{2}^{2}$ Médico asistencial, Servicio Pediatría, Hospital Universitario San José. Popayán, Colombia.

${ }^{3}$ Médico, Traumatólogo y Ortopedista, profesor Departamento de Cirugía, Facultad de Ciencias de la Salud, Universidad del Cauca. Popayán, Colombia. ${ }^{4}$ Médico, MSc en Epidemiología, profesor auxiliar Facultad de Ciencias de la Salud, Universidad ICESI. Cali, Colombia.

${ }^{5}$ Médica, MSc en Biología, PhD en Genética de Poblaciones Humanas y Genética Forense, profesora titular Departamento de Pediatría, Facultad de Ciencias de la Salud, Universidad del Cauca. Popayán, Colombia.

Conflicto de intereses: los autores declaran que no tienen conflicto de intereses Medicina \& Laboratorio 2015; 21: 179-192

Módulo 19 (Investigación), número 33. Editora Médica Colombiana S.A. $2015^{\odot}$

Recibido el 16 de marzo de 2015; aceptado el 05 de abril de 2015
} 
Colombia) between 2013 and 2014. Material and methods: an observational cohort study, closed and without comparison group was conducted. The zoledronic acid treatment was conducted quarterly for one year and during this time the positive and adverse effects of treatment were determined. Results: six patients had a diagnosis of osteogenesis imperfecta, of which one was excluded because it began surgical treatment, and five of them started treatment with zoledronic acid; subsequently, one patient voluntarily withdrew from the study found. From the four patients who completed one year of treatment with zoledronic acid, two not showed fractures during it and the other two each presented one fracture. Bone mineral density of the lumbar spine and femoral showed improvement; having two patients in the normal range at three months of treatment ends. According to the visual analogue scale, bone pain intensity at baseline ranged between 5 and 8 , and at the end between 0 and 2. Regarding functional limitation were not found significant changes nor adverse effects were found throughout follow-up. Conclusions: therapy with intravenous zoledronic acid in patients with osteogenesis imperfecta improves the course of disease independent of severity.

Keywords: osteogenesis imperfecta, biphosphonates, bone fractures, child.

Garcés-Constaín CE, Beltrán-Zúñiga E, Concha-Sandoval JM, Ariza-Araújo YJ, Acosta-Aragón MA. Efecto del ácido zoledrónico en pacientes pediátricos con diagnóstico clínico de osteogénesis imperfecta: un estudio de cohorte. Medicina \& Laboratorio 2015; 21 : 179-192.

$\mathrm{L}^{2}$ a osteogénesis imperfecta, también conocida como la enfermedad del hueso frágil o de los huesos de cristal, corresponde a un grupo heterogéneo de desórdenes congénitos caracterizados por presentar fragilidad del hueso debido a mutaciones en los genes que codifican para las cadenas polipeptídicas alfa- I y alfa-2 del procolágeno tipo I (COLIA I y COL IA2), generadas de forma hereditaria o por neo-mutación cromosómica. Estos genes se encuentran localizados en los cromosomas 7 y 17, respectivamente [1-3].

La incidencia de la osteogénesis imperfecta varía según la literatura; se han descrito cifras desde un caso por cada 5.000 hasta 70.000 nacidos vivos [4,5]. Es considerada como una de las 7.000 enfermedades «raras» que afectan a más de veinticinco millones de personas y sus familias [6], definidas así por presentar prevalencias de 200.000 o menos casos en los Estados Unidos [7]. En el contexto latinoamericano existen diferentes entidades dedicadas al estudio de la enfermedad y a brindar una mejor calidad de vida a los niños y adolescentes que la padecen, como la Fundación Ecuatoriana de Osteogénesis Imperfecta (FEOI) que atiende actualmente a 75 pacientes con osteogénesis imperfecta de escasos recursos económicos en todo Ecuador [8]. Hasta nuestro conocimiento, en Colombia aún no hay datos claros sobre la prevalencia de esta enfermedad [9-1 I].

La osteogénesis imperfecta se divide en cuatro grupos (I-IV) de acuerdo a la clasificación elaborada por Sillence y colaboradores (1979) [3] y que hasta el momento es la más utilizada. Las formas tipo I y tipo IV corresponden a las más leves de la enfermedad y que mayor número de casos aportan, caracterizadas por la ausencia de antecedentes familiares y un cuadro clínico de fracturas no traumáticas. De manera adicional Glorieux y colaboradores (2008) [12] describieron los tipos V, VI y VII de acuerdo a ciertas características histológicas y clínicas.

De manera general, la osteogénesis tipo I es la forma más común y leve de la enfermedad; no es deformante y los pacientes presentan talla normal, escleras azules y poca frecuencia de dentinogénesis imperfecta. Las fracturas no ocurren al nacimiento y disminuyen en número al llegar a la pubertad [13]. La tipo Il es la forma de osteogénesis imperfecta más severa, la cual resulta en la muerte del paciente durante el período perinatal debido a la presencia de múltiples fracturas intrauterinas costales y de huesos largos, deformidades esqueléticas severas e insuficiencia respiratoria [14]. La tipo III es otra de las formas más severas; sin embargo, es compatible con la vida después del período perinatal, ocurriendo deformidades 
óseas progresivas que inician al nacimiento. Las fracturas ocurren in útero y son comunes durante el crecimiento, generando talla baja y confinamiento a una silla de ruedas durante toda la vida [15]. La tipo IV tiene la presentación clínica más diversa, con fenotipo variable desde formas leves a severas [12,13].

El diagnóstico de osteogénesis imperfecta se fundamenta en los hallazgos clínicos y radiológicos; también se utilizan otros recursos como la evaluación de la densidad mineral ósea, marcadores bioquímicos de reabsorción ósea y estudios genéticos [16-19]. El tratamiento actual incluye intervenciones quirúrgicas para la estabilización intramedular y colocación de prótesis, y la terapia farmacológica con calcio, fluoruros y calcitonina, que se encuentran en desuso debido a que sus resultados clínicos no son muy favorables y no modifican la fragilidad intrínseca generada por esta patología [20]. Además, se utiliza la hormona de crecimiento que estimula el metabolismo óseo, y los bifosfonatos, entre los cuales se encuentra el ácido zoledrónico, cuyo uso ha sido aprobado para el tratamiento de la osteoporosis debido a que actúa sobre el hueso inhibiendo la acción de los osteoclastos, evitando la resorción ósea, lo que favorece el aumento de la densidad del hueso y, por lo tanto, disminuye el riesgo de fracturas [2I].

Desde 1987 se ha encontrado, de forma experimental, cómo los bifosfonatos modifican la calidad de vida de los pacientes con osteogénesis imperfecta, disminuyendo el número de fracturas asociadas y las complicaciones secundarias, y mejorando la densidad mineral ósea, lo que aumenta de manera significativa las probabilidades de éxito con el tratamiento quirúrgico [22]. Este efecto se ha atribuido a su capacidad antiresortiva, ya que se unen a componentes minerales expuestos por los osteoclastos durante el proceso de resorción ósea y son reabsorbidos por estas células junto con el hueso durante los ciclos tardíos de remodelación ósea, inhibiendo así la función osteoclástica hasta inducir la apoptosis de estas células [23].

Los bifosfonatos nitrogenados como el pamidronato y el ácido zoledrónico tienen efectos clínicos y paraclínicos más significativos sin generar efectos adversos importantes, con evidencia que se soporta en pacientes pediátricos con osteogénesis imperfecta sometidos a este tratamiento, un incremento en la densidad mineral ósea, disminución de la tasa de fracturas, mejoría de la deambulación y de los marcadores bioquímicos de remodelación ósea, así como la disminución del dolor [20,22-25]. Además, se ha observado en los estudios realizados en los últimos años mejores resultados con el uso de ácido zoledrónico que con pamidronato, con un esquema de aplicación más conveniente [26]. Con base en lo anterior el objetivo de este trabajo fue determinar el efecto del tratamiento con ácido zoledrónico endovenoso en un grupo de pacientes pediátricos con osteogénesis imperfecta atendidos en el Hospital Universitario San José (Popayán, Colombia) durante 2013 y 2014.

\section{Materiales y métodos}

\section{Tipo y población de estudio}

Se realizó un estudio observacional tipo cohorte de carácter cerrado y sin grupo de comparación. La selección de este diseño se realizó teniendo en cuenta que la administración del ácido zoledrónico es poco frecuente en los pacientes pediátricos y que la osteogénesis imperfecta es una enfermedad de baja incidencia y de esporádica ocurrencia. Se tomó como muestra todos los casos con diagnóstico clínico de osteogénesis imperfecta en edad pediátrica a los que se les realizó tratamiento con ácido zoledrónico en el Centro de Infusión Pediátrica del Hospital Universitario San José (Popayán, Colombia) entre 2013 y 2014.

La administración del medicamento se realizó según las indicaciones terapéuticas de las guías actuales de manejo de la enfermedad, momento a partir del cual se inició el seguimiento de los pacientes para 
determinar el desarrollo de los eventos de interés: efectos positivos esperados del medicamento como el aumento de la densidad mineral ósea y las variables clínicas relacionadas (número de fracturas, dolor y limitación funcional), así como los efectos negativos (reacciones adversas reportadas por la literatura). La recolección de información fue de manera prolectiva (simultáneamente a la administración del medicamento). Se diseñaron protocolos operativos estandarizados para la obtención de la información pertinente a partir de fuentes primarias: los propios pacientes y los cuidadores de estos.

\section{Criterios de inclusión}

Pacientes pediátricos (entre un mes y 18 años de vida) con diagnóstico clínico de osteogénesis imperfecta, tratamiento con ácido zoledrónico entre el año 2013 y 2014 con una duración mínima de 12 meses, no haber recibido tratamiento con otro bifosfonato previo al inicio de la terapia con ácido zoledrónico y firma del consentimiento informado por parte del padre o adulto responsable.

\section{Criterios de exclusión}

Pacientes que no cumplen con los criterios de inclusión, que presenten una condición clínica que contraindique el uso del ácido zoledrónico (p. ej. función renal alterada), que hayan cambiado de lugar de residencia por fuera del departamento del Cauca, Colombia, durante el período de seguimiento, o inicio del tratamiento de remodelación ósea por traumatología.

\section{Variables de estudio}

Dentro de las variables evaluadas se encuentran:

$\rightarrow$ Sociodemográficas: edad, género, procedencia, estrato socioeconómico.

$\rightarrow$ Clínicas: evaluadas según el momento del estudio:

$\rightarrow$ Al inicio: antecedentes familiares de osteogénesis imperfecta o de fracturas a repetición, edad al momento del diagnóstico, edad de inicio del tratamiento, edad de primera fractura, número de fracturas e intervenciones quirúrgicas previas al inicio del tratamiento.

$\rightarrow$ Al inicio y durante el seguimiento: antecedentes familiares de osteogénesis imperfecta o de fracturas a repetición, edad al momento del diagnóstico, edad de inicio del tratamiento, edad de primera fractura, número de fracturas e intervenciones quirúrgicas previas al inicio del tratamiento.

$\rightarrow \mathrm{Al}$ inicio y durante el tratamiento: peso $(\mathrm{Kg})$, talla (cm), Índice de Masa Corporal (IMC) con ubicación en tablas estándar de la Organización Mundial de la Salud, número de fracturas durante el tratamiento, dolor percibido medido por la escala de dolor visual análoga, validada con dibujos faciales, o por la escala adaptada de FLACC (del inglés, Face, Legs, Activity, Cry, Consolability) en los pacientes menores de cuatro años, los cuales no realizaban fácilmente la identificación de un dibujo facial representativo del dolor, grado de limitación funcional medido por la escala de Bleck y efectos adversos durante la infusión y entre infusiones.

$\rightarrow$ De laboratorio o paraclínicas: medidas según el momento del estudio:

$\rightarrow$ Al inicio y al final del tratamiento: densidad mineral ósea en vértebras lumbares y cuello femoral.

$\rightarrow \mathrm{Al}$ inicio y durante el seguimiento: creatinina sérica, nitrógeno ureico en sangre, hemograma, calcio y fósforo urinarios y séricos. 


\section{Administración del ácido zoledrónico}

De acuerdo a lo establecido en el protocolo del Centro de Infusión Pediátrica, previo inicio del tratamiento, los pacientes y sus padres fueron informados acerca de los efectos adversos potenciales secundarios al uso del ácido zoledrónico más comunes, tales como hipocalcemia y fiebre, así como las complicaciones raras descritas en adultos (p. ej. osteonecrosis de la mandíbula).

El medicamento se aplicó a dosis de $0,05 \mathrm{mg} / \mathrm{Kg}$ mediante infusión intravenosa durante 60 minutos por dos días consecutivos, diluido en $50 \mathrm{~mL}$ de solución salina normal al 0,9\%, con una dosis máxima de $2 \mathrm{mg}$ por infusión, con periodicidad trimestral durante las cinco primeras dosis y luego semestral. Posterior a cada dosis se brindó analgesia con acetaminofén o ibuprofeno por dos días según la necesidad y aporte de calcio por la primera semana. En pacientes menores de un año la dosis usada fue de $0,025 \mathrm{mg} / \mathrm{Kg}$.

\section{Recolección y análisis de datos}

Se realizó una evaluación periódica de los pacientes incluidos en el estudio, inicialmente para conocimiento de la historia clínica previa, los datos sociodemográficos y los antecedentes de importancia, y posteriormente, cinco evaluaciones más, dos de ellas realizadas por un médico especialista en traumatología y ortopedia.

Para la recolección de la información se empleó un instrumento elaborado por los investigadores y los datos obtenidos fueron consignados en una base de datos en Excel. Posteriormente, se realizó un análisis descriptivo y gráfico utilizando para las variables cuantitativas medidas de tendencia central y dispersión (media y desviación estándar) y para las variables cualitativas frecuencias absolutas y proporciones.

\section{Resultados}

\section{Casos con osteogénesis imperfecta}

Según el reporte del Departamento de Estadística del Hospital Universitario San José (Popayán, Colombia), se registraron 14 casos de osteogénesis imperfecta desde el año 201 I. El código CIE IO consultado fue Q780. De estos 14 casos fue posible contactar a seis.

Los pacientes contactados contaban con diagnóstico clínico de osteogénesis imperfecta realizado en el Hospital Universitario San José por un médico especialista en genética clínica, y de edad entre uno y 14 años, quienes no habían recibido tratamiento previo con otro bifosfonato. Los padres de estos seis pacientes firmaron el consentimiento informado de participación en el estudio para recibir infusión endovenosa con ácido zoledrónico entre los años 2013 y 20 I 4, con duración mínima de la infusión de 12 meses, período durante el cual se realizó el seguimiento y la aplicación del medicamento de forma trimestral.

Dos de los pacientes fueron excluidos, uno de ellos debido a que inició tratamiento quirúrgico ortopédico que contraindicó el uso de bifosfonatos durante el mismo y otro presentó una censura en el sexto mes de seguimiento, posterior al segundo ciclo de infusión, y se retiró de forma voluntaria pues el padre no autorizó continuar con la aplicación del medicamento (véase figura I). 
Búsqueda inicial: 2011-2014

Departamento de Estadística del Hospital Universitario San José, Popayán, Colombia

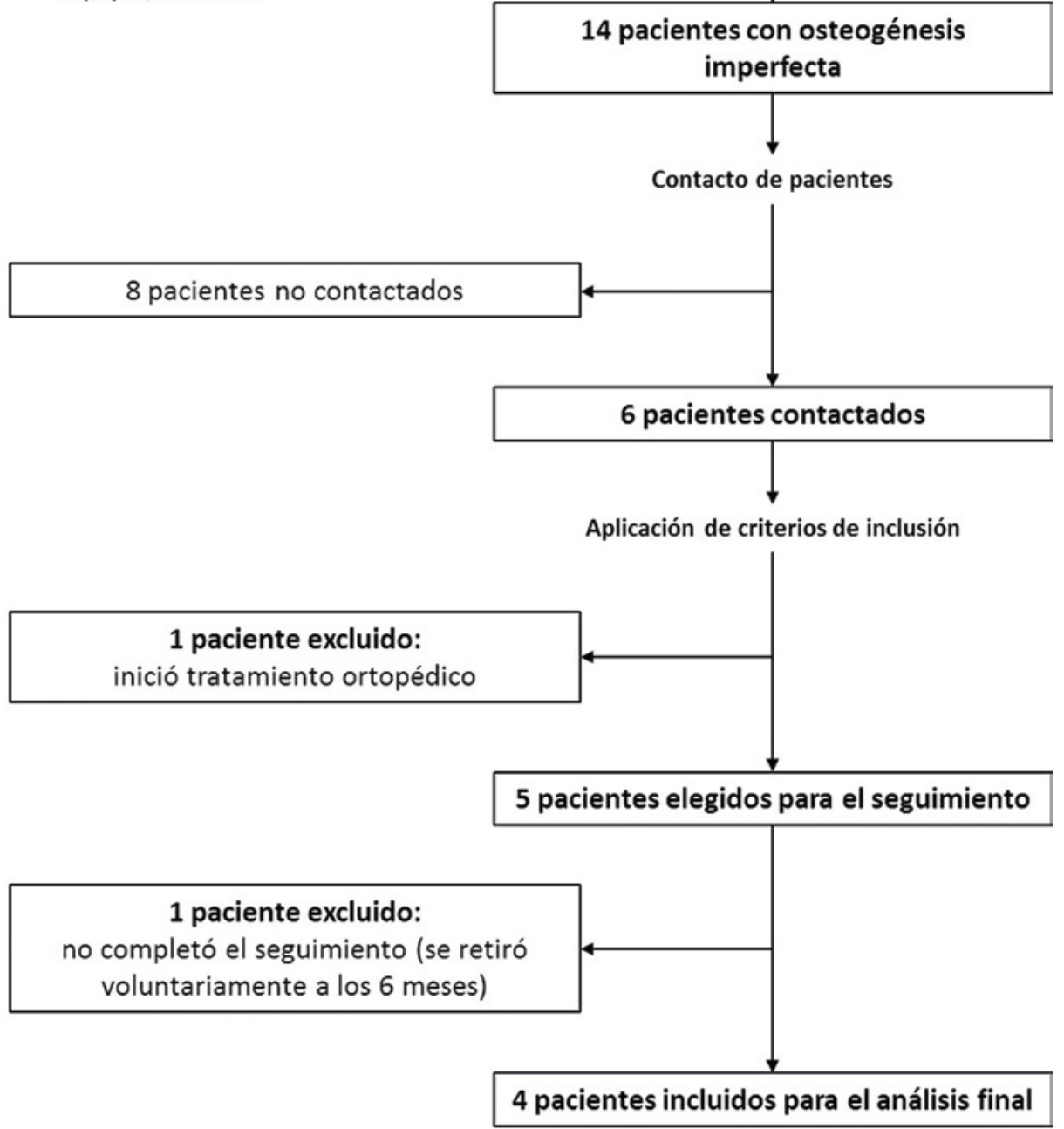

Figura I. Diagrama de la inclusión, exclusión y seguimiento de los participantes del estudio.

\section{Características sociodemográficas y clínicas de los pacientes}

La edad promedio de los pacientes incluidos fue de ocho años, tres de ellos del género femenino y dos del masculino, tres originarios del área rural: dos de Argelia y uno de El Bordo (municipios del departamento del Cauca, Colombia) y dos procedentes de Popayán (capital del departamento del Cauca, Colombia). Dos de los participantes presentaban antecedentes familiares de osteogénesis imperfecta en primer grado de parentesco; sin embargo, no se documentó en ninguno de los casos historia de consanguinidad entre los padres. Todos los pacientes se encontraban afiliados al régimen subsidiado de seguridad en salud. 
La edad promedio de diagnóstico fue de 6,4 años, dos de ellos con sospecha diagnóstica de displasia esquelética a los cinco y seis meses de edad gestacional, respectivamente, y uno de estos con diagnóstico clínico de osteogénesis imperfecta en la edad neonatal. La totalidad de los casos no había recibido tratamiento farmacológico con bifosfonatos previo al estudio. Cuatro pacientes presentaban antecedentes de fracturas a repetición (entre tres y 18 fracturas), tres de los cuales requirieron tratamiento quirúrgico correctivo por parte de traumatología. La edad promedio a la que se presentó la primera fractura fue 4,3 años, ocurriendo en uno de los casos en la edad neonatal. Dos pacientes presentaban formas leves a moderadas de la enfermedad mientras que los otros tres mostraron formas severas.

De los cuatro pacientes que completaron el año de tratamiento con ácido zoledrónico dos no presentaron fracturas durante este período, los otros dos presentaron una fractura cada uno, ambos durante el segundo ciclo de tratamiento. El paciente que se retiró del estudio después del segundo ciclo no había presentado ninguna fractura hasta el momento en que finalizó su participación. En la tabla I se presentan de forma detallada las características de los pacientes incluidos en este estudio.

\begin{tabular}{|c|c|c|c|c|c|}
\hline Parámetro & Paciente I & Paciente 2 & Paciente 3 & Paciente 4 & Paciente 5 \\
\hline Edad (años) & 7 & II & । & 7 & 14 \\
\hline Género & Masculino & Femenino & Femenino & Femenino & Masculino \\
\hline Origen & Rural & Rural & Urbano & Rural & Urbano \\
\hline Sistema de salud & Subsidiado & Subsidiado & Subsidiado & Subsidiado & Subsidiado \\
\hline Antecedente familiar & $\mathrm{Si}$ & $\mathrm{Si}$ & No & No & No \\
\hline Edad diagnóstico (años) & 6 & 11 & 0 & 2 & 13 \\
\hline Consanguinidad de los padres & No & No & No & No & No \\
\hline $\begin{array}{l}\text { Tratamiento farmacológico } \\
\text { previo }\end{array}$ & No & No & No & No & No \\
\hline Número de fracturas previas & 3 & 0 & 5 & 18 & 4 \\
\hline $\begin{array}{l}\text { Edad de la primera fractura } \\
\text { (años) }\end{array}$ & 6 & No aplica & 0 & 0 & II \\
\hline Manejo quirúrgico & $\mathrm{Si}$ & No & No & $\mathrm{Si}$ & $\mathrm{Si}$ \\
\hline
\end{tabular}

En cuanto a los parámetros antropométricos en la valoración inicial los cinco pacientes se encontraban en el rango de talla baja desproporcionada característica, sin cambios significativos hasta la valoración final. Con respecto al peso e Índice de Masa Corporal (IMC) al inicio del estudio se encontró sobrepeso en un paciente y obesidad en otro de ellos, y en la evaluación final se documentó sobrepeso en dos pacientes y obesidad en uno, lo que muestra una tendencia al aumento del IMC durante el seguimiento en estos pacientes.

Respecto a la intensidad del dolor óseo reportada en la escala visual analógica al inicio del estudio los cuatro pacientes que completaron el tratamiento reportaron valores entre 5 y 8 . Después de cuatro ciclos de infusión con ácido zoledrónico reportaron valores entre 0 y 2. La limitación funcional evaluada con la escala de Bleck se comportó de la siguiente manera: dos pacientes se mantuvieron funcionales con el máximo valor de la escala (9), uno mejoró pasando de un puntaje de I a 4 y el otro no mejoró la puntuación permaneciendo en I. 


\section{Características de laboratorio o paraclínicas de los pacientes}

De los cuatro pacientes que completaron el estudio se encontró un valor promedio de densidad mineral ósea previa al inicio del tratamiento a nivel de la columna lumbar de 0,386, siendo clasificados todos ellos en el rango de osteopenia y, a nivel femoral de 0,397, siendo clasificados tres de los pacientes en rango de osteopenia y uno en rango de osteoporosis. Durante la evaluación a los tres meses de terminado el tratamiento la densidad mineral ósea fue determinada únicamente en tres de los cuatro pacientes, encontrando un valor promedio a nivel de columna lumbar de 0,609 y a nivel femoral de 0,657, siendo clasificados dos de los pacientes en rango de osteopenia y uno en rango de normalidad para ambos parámetros (véase figuras 2 y 3).

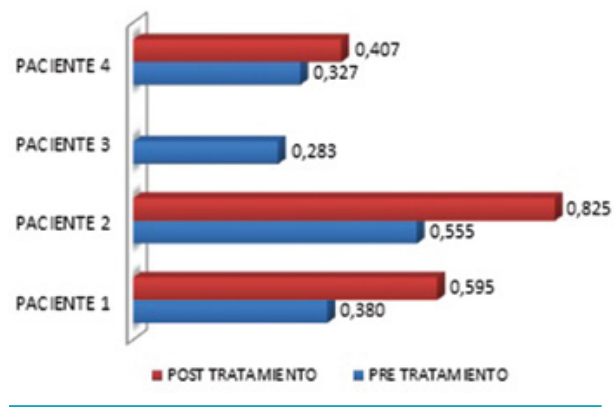

Figura 2. Densidad mineral ósea columna lumbar $\left(\mathrm{g} / \mathrm{cm}^{2}\right)$

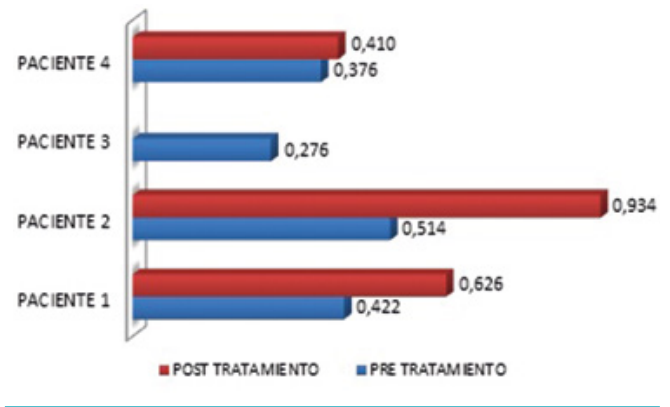

Figura 3. Densidad mineral ósea femoral $\left(\mathrm{g} / \mathrm{cm}^{2}\right)$

No se documentaron alteraciones significativas de la función renal ni en los electrolíticas durante el seguimiento, ni cambios patológicos en los hemogramas. En cuanto a los efectos adversos no se registró ninguno severo o moderado durante el seguimiento. Todos los pacientes describieron presencia de dolor óseo en las 48 horas siguientes a la aplicación de alguna de las infusiones del primer y segundo ciclo. Este síntoma no fue reportado en los dos ciclos siguientes. Sólo un participante presentó fiebre posterior a la segunda infusión que se controló con el uso de acetaminofén vía oral.

\section{Evolución de la enfermedad en los pacientes tratados con ácido zoledrónico}

Puesto que el número de participantes fue pequeño y la información sobre la evolución de la enfermedad durante la administración del medicamento es de gran importancia para el conocimiento de esta condición, a continuación se presenta la descripción detallada de cada uno de los participantes:

$\rightarrow$ Paciente I: paciente masculino de siete años de edad, de origen rural, etnia mestiza con predominio nativo americano, con antecedente familiar de osteogénesis imperfecta, diagnosticado a los seis años de edad, sin historia de consanguinidad entre sus padres. Historia personal de tres fracturas previas al inicio del tratamiento con ácido zoledrónico, todas dentro de los 12 meses anteriores a la intervención, y con requerimiento de manejo quirúrgico. El paciente cumplió con los tiempos de evaluación y aplicación del medicamento acorde a lo establecido en el protocolo. El puntaje inicial del dolor percibido fue de 5, con una disminución de los puntos en la escala durante el seguimiento y un valor en la evaluación final de I. La limitación funcional en el inició y al final de tratamiento permaneció en 9, el máximo valor para clasificar al paciente como funcional, evidenciado por la marcha independiente sin necesidad de apoyo en muletas (véase figura 4). La incidencia de fracturas por año, en el año 
anterior al inicio del tratamiento, fue de tres (3 fracturas/año) y para el año de tratamiento no presentó ninguna fractura (0 fracturas/año).

$\rightarrow$ Paciente 2: paciente femenina de 1 I años de edad, de origen rural, etnia mestiza con predominio nativo americano y antecedente familiar de osteogénesis imperfecta, tía en primer grado del paciente I. Fue diagnosticada a los II años de edad, sin historia de fracturas previas ni de consanguinidad entre sus padres. Historia de dolor óseo de predominio en miembros inferiores y pelvis, en ocasiones incapacitante, asociado a deformidad en varo de miembros inferiores. La paciente cumplió con los tiempos de evaluación y aplicación del ácido zoledrónico. El puntaje inicial de dolor percibido fue de 7, con una disminución de los puntos en la escala durante el seguimiento y un valor en la evaluación final de I. La limitación funcional evaluada con la escala de Bleck durante todo el período de seguimiento permaneció en 9, el máximo valor para clasificar al paciente como funcional, evidenciado por la marcha independiente sin necesidad de apoyo en muletas (véase figura 5). Esta paciente, como se mencionó previamente, no presentó fracturas previas al inicio del tratamiento ni durante el mismo ( 0 fracturas/año).

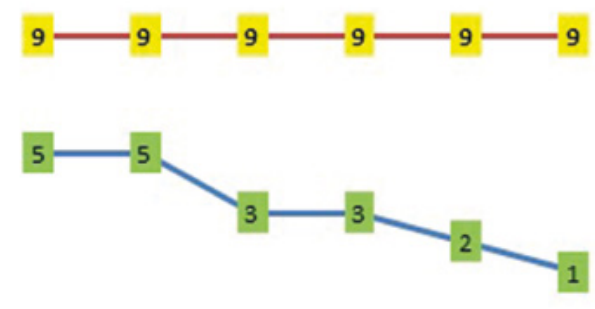

INICIAL CICLO 1 CICLO 2 CICLO3 CICLO 4 FINAL

Figura 4. Dolor escala visual analógica (línea azul) y limitación funcional escala de Bleck (línea roja) en el paciente I.

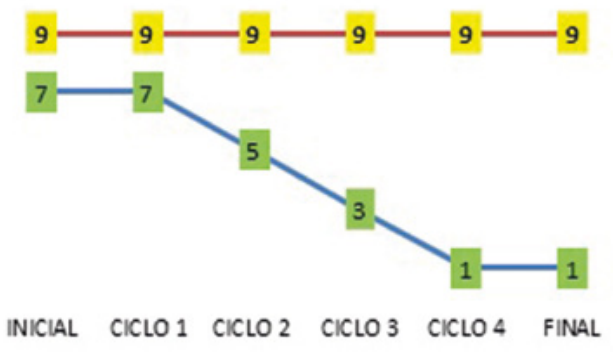

Figura 5. Dolor escala visual analógica (línea azul) y limitación funcional escala de Bleck (línea roja) en la paciente 2.

$\rightarrow$ Paciente 3: paciente femenina de un año de edad, origen urbano, etnia mestiza con predominio nativo americano, sin antecedente familiar de osteogénesis imperfecta ni historia de consanguinidad entre sus padres. La paciente presentó sospecha diagnóstica de displasia esquelética antenatal a los cinco meses de edad gestacional y diagnóstico clínico en la edad neonatal, período en el que presentó cuatro fracturas, la primera a los dos días de vida, y una quinta fractura a los nueve meses. La paciente cumplió con los tiempos de evaluación y aplicación del medicamento. El puntaje inicial del dolor percibido fue de 7, con una disminución de los puntos en la escala durante el seguimiento y un valor en la evaluación final de 0 . La limitación funcional evaluada inicialmente fue de I debido a que la paciente aún no caminaba, encontrando un aumento de los puntos durante el seguimiento y un valor final de 4, evidenciado porque la paciente al final del seguimiento camina en casa con apoyo, lo que la clasifica como funcional en esta escala (véase figura 6). La incidencia de fracturas por año, en el año anterior al inicio del tratamiento, fue de cinco ( 5 fracturas/año) y durante el año de tratamiento de uno ( 1 fractura/año).

$\rightarrow$ Paciente 4: paciente femenina de siete años de edad, etnia afrodescendiente, origen rural, sin antecedente familiar de osteogénesis imperfecta ni de consanguinidad entre los padres, y diagnóstico clínico a los dos años de edad. Historia de 19 fracturas desde el nacimiento, la primera a los dos meses de vida, ninguna en el año previo al inicio del tratamiento con ácido zoledrónico. La paciente no cumplió con los tiempos de evaluación y aplicación del medicamento debido a dificultades con los trámites administrativos para la autorización de las infusiones, la toma de los paraclínicos de seguimiento y la 
gestión por parte de la madre para los mismos, produciéndose un retraso de tres meses para la aplicación de la segunda infusión y de dos meses para la tercera. El puntaje inicial del dolor percibido fue de 5, con una disminución de los puntos en la escala durante el seguimiento y un valor en la evaluación final de 2. La limitación funcional evaluada inicialmente fue de I, valor que se mantuvo durante todo el período de seguimiento debido a que la paciente no puede caminar, lo que la clasifica como no funcional (véase figura 7). La incidencia de fracturas por año, en el año anterior al inicio de tratamiento, fue de cero (0 fracturas/año) y durante el año de tratamiento de uno (I fractura/año). De todos los pacientes evaluados es la que más fracturas había presentado desde su nacimiento, con un total de 19 , teniendo en promedio tres a cuatro fracturas por año en los primeros años de vida.

El consolidado de la incidencia de fracturas patológicas en el año previo al inicio del tratamiento fue de nueve por cuatro personas por año (2,25 fracturas/persona/año), y durante el año de tratamiento de dos por cuatro personas por año (0,5 fracturas/persona/año), lo que quiere decir que la velocidad de fractura en el año previo al tratamiento es 4,5 veces mayor que la velocidad de fractura durante el año de tratamiento, es decir, la velocidad de fractura disminuyó en un $78 \%$ con el tratamiento (véase figura 8).

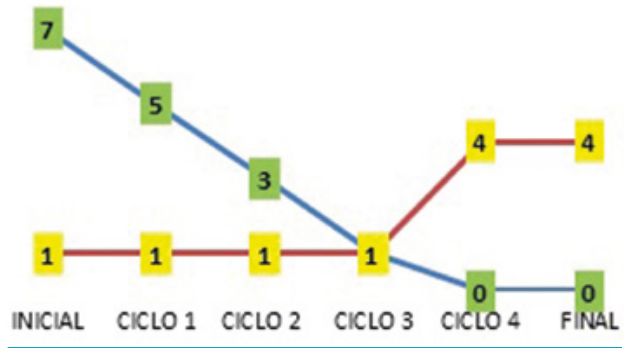

Figura 6. Dolor escala visual analógica (línea azul) y limitación funcional escala de Bleck (línea roja) en la paciente 3.

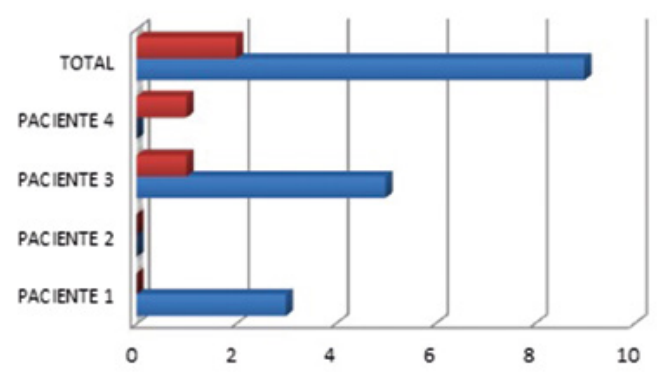

\# FRACTURAS DURANTE TRATAMIENTO $\equiv$ " FRACTURAS 1 AÑ̃O PRE TRATAMIENTO

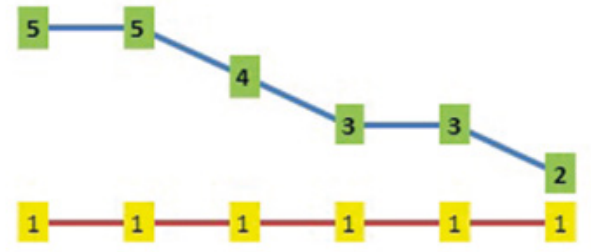

INICIAL CICLO 1 CICLO 2 CICLO3 CICLO4 FINAL

Figura 7. Dolor escala visual analógica (línea azul) y limitación funcional escala de Bleck (línea roja) en la paciente 4.
Figura 8. Número de fracturas de los pacientes un año antes del tratamiento y durante el tratamiento.

\section{Discusión}

La osteogénesis imperfecta, como grupo heterogéneo de desórdenes con la característica común de presentar fragilidad congénita del hueso causada por mutaciones en los genes que codifican para el procolágeno tipo I (COLIAI y COLIA2), es una condición que requiere idealmente diagnóstico molecular, el cual no estuvo disponible para los pacientes de nuestro estudio. Una posible solución definitiva a la susceptibilidad de fracturas en estos pacientes sería la intervención en el defecto basal por medio de terapia génica, con la cual no se cuenta en la actualidad [I-3]. 
En nuestro medio la forma de identificar a los pacientes con osteogénesis imperfecta es mediante diagnóstico clínico teniendo en cuenta las características de la enfermedad. En este estudio se encontraron dos pacientes con diagnóstico probable de osteogénesis imperfecta tipo III, dada la presencia de manifestaciones desde la edad neonatal o el período de lactante menor y la severidad del cuadro desde edades tempranas, y otros dos con diagnóstico probable de osteogénesis imperfecta tipo l, la forma más común de presentación caracterizada por manifestaciones leves o menos severas y una herencia autosómica dominante, dada la presencia de características sugestivas de la enfermedad en las cuatro generaciones antecesoras a dichos pacientes. Para el otro paciente no fue posible determinar el tipo de osteogénesis imperfecta, aunque es una forma con características de severidad. Es importante hacer énfasis en esta clasificación y características debido a que estas condiciones intrínsecas de cada paciente no son modificables ni susceptibles a la intervención, pero pueden influenciar en la diferencia entre los resultados obtenidos en cada caso y, por supuesto, en la severidad de la presentación $[12,13,15]$.

Teniendo en cuenta que hasta el momento no es posible modificar la susceptibilidad genética ni la severidad de la presentación del cuadro clínico según el tipo de mutación como alternativa terapéutica, una opción viable es la utilización de múltiples agentes farmacológicos que influyen en el proceso de remodelación ósea, en particular el uso de bifosfonatos nitrogenados como el ácido zoledrónico, que combate la actividad osteoclástica mediante la inducción de apoptosis o inhibición de su funcionalidad, y ha demostrado resultados favorables en estudios realizados en pacientes pediátricos, representada por mejoría de la densidad mineral ósea y disminución tanto en el número de fracturas patológicas como del dolor y la limitación funcional asociados [20,22].

En nuestro estudio se administró una infusión de ácido zoledrónico $(0,05 \mathrm{mg} / \mathrm{Kg})$ de forma trimestral para determinar durante el período de seguimiento los cambios en los parámetros de densidad mineral ósea, fracturas patológicas, dolor y limitación funcional. Durante el estudio se tuvo en cuenta la presencia de posibles factores confusores que pueden influenciar en estos resultados, disminuyendo la densidad mineral ósea ya alterada por la condición basal, como son la disminución en la actividad física en estos pacientes, el estado nutricional inadecuado que puede alterar los minerales, hormonas o electrolitos fundamentales para el metabolismo óseo y la exposición insuficiente a la luz solar necesaria para la absorción de la vitamina D. No obstante, durante todo el seguimiento no hubo cambios significativos o intervenciones que modificaran de forma importante ninguno de estos factores, lo que permite considerar que los resultados obtenidos en nuestro estudio son probablemente atribuibles a la única intervención diferente realizada que fue recibir el ácido zoledrónico.

Los resultados del presente estudio sugieren que el tratamiento con ácido zoledrónico endovenoso tiene efectos benéficos en la salud ósea de los niños y adolescentes con osteogénesis imperfecta, dado el aumento en la densidad mineral ósea a nivel lumbar y femoral, con cambios significativos posterior al año de seguimiento, pasando el paciente, en algunos casos, de osteoporosis a osteopenia o de osteopenia a densidad mineral ósea normal. Estos hallazgos son similares a los demostrados por Vuorimies y colaboradores (20I I) [27], quienes utilizaron iguales dosis de ácido zoledrónico por vía intravenosa en pacientes con osteogénesis imperfecta de uno a 16 años de edad y encontraron que la densidad mineral ósea de la columna lumbar aumentó significativamente. De igual manera, Barros y colaboradores (20 I2) [26] utilizaron el ácido zoledrónico en comparación con otro bifosfonato, el pamidronato, y encontraron un aumento significativo de la densidad mineral ósea con ambos medicamentos; sin embargo, los resultados fueron más favorables con el ácido zoledrónico.

En este trabajo se evidenció que la administración cíclica de ácido zoledrónico también genera un impacto positivo en los pacientes en cuanto a la disminución en la incidencia de fracturas, de nueve en el año anterior al inicio de la intervención a dos en el año de tratamiento de manera significativa, lo que se correlaciona con los hallazgos de Lazala y Solaque (2009) [28] en niños de Bogotá, Colombia, tratados con 
pamidronato, cuya incidencia de fracturas disminuyó de 7,9 al inicio del tratamiento a 1,8 después de este. Además, los autores demuestran una importante disminución del dolor y aumento de la mineralización ósea que mejora así su calidad de vida, relacionado con la disminución en la necesidad de intervenciones quirúrgicas y estancias hospitalarias.

El dolor óseo asociado a la enfermedad también se redujo de manera importante en los pacientes tratados en este estudio, como probable consecuencia del aumento en la densidad ósea y del engrosamiento cortical, que disminuyen la presencia de microfracturas y fracturas patológicas [9-1 I]. En cuanto a la limitación funcional medida con la escala de Bleck no se encontraron en la mayoría de pacientes cambios significativos entre el puntaje al inicio del tratamiento y al final de este, con el inconveniente de que esta la única escala validada para evaluar la funcionalidad en pacientes con osteogénesis imperfecta, pero presenta parámetros limitados para la estimación de estas características, especialmente en pacientes pediátricos, quienes se encuentran continuamente en crecimiento y desarrollo [29].

Los parámetros antropométricos propuestos a evaluar al inicio del estudio presentaron una gran limitación en la obtención de datos confiables y objetivos, debido a la postración de algunos de estos pacientes, lo que hizo difícil especialmente la medición precisa de la talla y el peso. Con los datos recolectados se encontró tendencia al sobrepeso y aumento mínimo, pero progresivo, del Índice de Masa Corporal (IMC) en todos los pacientes, lo cual podría estar relacionado con la menor actividad física y el aumento de la densidad mineral ósea. Otra de las limitaciones fue la censura presentada por una de las pacientes en cuanto al cumplimiento en la aplicación del medicamento, con retraso en la administración del mismo entre dos a tres meses, en la que se observó un menor aumento de la densidad mineral ósea con respecto a los demás pacientes. Esta condición podría atribuirse a la irregularidad de las infusiones y también a la severidad del tipo de osteogénesis imperfecta [14,15], como ocurrió en dicha paciente.

En este estudio ningún paciente presentó efectos adversos severos o moderados tras el tratamiento con ácido zoledrónico, y aquellos que los presentaron los manifestaron durante las 48 horas siguientes a la infusión, caracterizados principalmente por dolor óseo o fiebre de fácil resolución, especialmente en las primeras dos aplicaciones del medicamento, sin observarse alteración en la función renal o en los electrolitos, de acuerdo a lo descrito en la literatura [I-3]. En cuanto a los demás paraclínicos evaluados, principalmente las pruebas serológicas para evaluar metabolismo óseo, no fue posible generar comparaciones o estimaciones de los cambios generados, debido a que en la mayoría de pacientes no fueron autorizados estos exámenes por las instituciones prestadoras de salud a las que pertenecían o no fueron realizados en su totalidad o de manera oportuna para ser comparables.

En la figura 9 se presenta un mapa conceptual en el que se plantea la explicación del efecto de los bifosfonatos en el curso de la osteogénesis imperfecta.

\section{Conclusiones}

La terapia con ácido zoledrónico endovenoso en pacientes con diagnóstico clínico de osteogénesis imperfecta ofrece una forma efectiva de alterar favorablemente el curso natural de la enfermedad, independientemente de la severidad de la presentación clínica [20-22,30], planteando un mayor beneficio en cuanto a los parámetros evaluados de densidad mineral ósea, fracturas patológicas, dolor y limitación funcional en las formas de osteogénesis imperfecta con menor severidad desde el inicio [23-25,3 I]. 


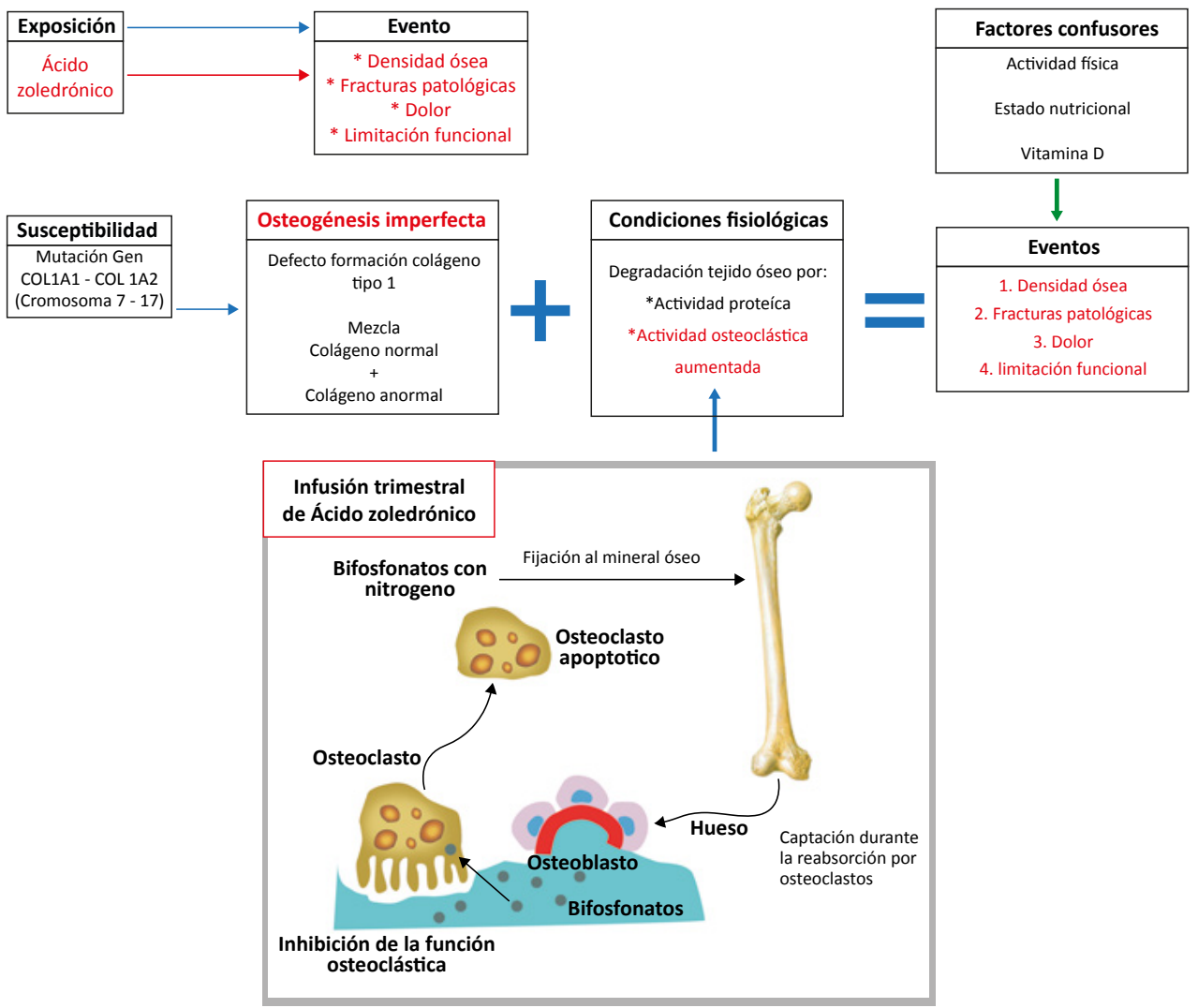

Figura 9. Mapa conceptual que explica el efecto de los bifosfonatos en el curso de la osteogénesis imperfecta. En más del 90\% de pacientes con la enfermedad se encuentra una mutación del gen COLIAI o COL IA2 (cromosomas 7 o I7), lo cual genera una formación defectuosa del colágeno tipo I en cuanto a cantidad o calidad, que se mezcla con el colágeno tipo I normal, generando inestabilidad funcional del mismo. En consecuencia, se encuentra tejido óseo fácilmente degradable, bien sea por la actividad lítica proteica u osteoclástica, estando aumentada está última, lo que genera una formación defectuosa de la matriz ósea y disminución de la mineralización ósea, que en consecuencia generaría disminución de la densidad mineral ósea y de forma secundaria la aparición de microfracturas, fracturas patológicas y dolor, y en última instancia limitación funcional, convirtiéndose así en un círculo vicioso. Dentro de este proceso hay factores confusores que también pueden afectar en diferente grado la densidad mineral ósea, como el grado de actividad física, el estado nutricional y la exposición a la luz solar (vitamina D). Información y gráfico tomados y modificados de «Controversia en relación con el uso de bifosfonatos en pacientes con enfermedad renal» por Restrepo-Valencia y Manjarrés-Iglesias, 2009, Acta Med Colomb, 34, p. 176-184. Copyright 201 I-20I 4 por Asociación Colombiana de Medicina Interna.

\section{Bibliografía}

I. Marini JC. Osteogenesis imperfecta: comprehensive management. Adv Pediatr 1988; 35: 39I-426.

2. Prockop DJ, Kivirikko KI. Heritable diseases of collagen. N Engl J Med 1984; 31 I: 376-386.

3. Sillence DO, Senn A, Danks DM. Genetic heterogeneity in osteogenesis imperfecta. J Med Genet 1979; 16: $101-116$.

4. Genetics Home Reference. Osteogenesis imperfecta. Maryland, Estados Unidos: U.S. National Library of Medicine®. 2013. Disponible: http://ghr.nlm.nih.gov/condition/ osteogenesis-imperfecta. Consultado: enero 2015
5. Beary JF, III, Chines AA. Osteogenesis imperfecta: Clinical features and diagnosis. Massachusetts,. Estados Unidos: UpToDate. 2015. Disponible: http://www.uptodate.com/contents/osteogenesis-imperfecta-clinicalfeatures-and-diagnosis. Consultado: enero 2015.

6. Genetic and Rare Diseases (GARD) Information Center. Genetic and Rare Diseases. Maryland, Estados Unidos: U.S. Department of Health \& Human Services, National Institutes of Health, National Center for Advancing Translational Sciences,. 2015. Disponible: https://rarediseases.info.nih.gov/gard/search-results. Consultado: enero 2015. 
7. Public Law 98-55I. Health Promotion and Disease Prevention Amendments of 1984. Octubre 30, 1984. Disponible: https://history.nih.gov/research/downloads/ PL98-55I.pdf. Consultado: enero 2015.

8. Fundación Ecuatoriana de Osteogénesis Imperfecta (FEOI). Disponible: http://feoi.wikta.com/index.php. Consultado: enero 2015.

9. Rauch F, Glorieux FH. Osteogenesis imperfecta. Lancet 2004; 363: 1377-1385.

10. Tosi LL. Osteogenesis imperfecta. Curr Opin Pediatr 1997; 9: 94-99.

II. Shapiro JR, Sponsellor PD. Osteogenesis imperfecta: questions and answers. Curr Opin Pediatr 2009; 21 : 709-7I6.

12. Glorieux FH. Osteogenesis imperfecta. Best Pract Res Clin Rheumatol 2008; 22: 85-100.

13. Semler O, Cheung MS, Glorieux FH, Rauch F. Wormian bones in osteogenesis imperfecta: Correlation to clinical findings and genotype. Am J Med Genet A 20I0; 152A: 1681-1687.

14. Madu AE, Olamijulo JA. Pregnancy complicated by a severe form of foetal osteogenesis imperfecta in a 17-year-old primigravida: case report and overview of literature. J Matern Fetal Neonatal Med 20 I3; 26: 703705.

15. Plotkin $\mathrm{H}$. Syndromes with congenital brittle bones. BMC Pediatr 2004; 4: 16

16. Whyte MP. Hypophosphatasia and the role of alkaline phosphatase in skeletal mineralization. Endocr Rev 1994; 15: 439-461.

17. Chines A, Boniface A, McAlister W, Whyte M. Hypercalciuria in osteogenesis imperfecta: a follow-up study to assess renal effects. Bone 1995; 16: 333-339.

18. Lund AM, Hansen M, Kollerup G, Juul A, Teisner B, Skovby F. Collagen-derived markers of bone metabolism in osteogenesis imperfecta. Acta Paediatr 1998; 87: $1131-1137$.

19. Rauch F, Travers R, Parfitt AM, Glorieux FH. Static and dynamic bone histomorphometry in children with osteogenesis imperfecta. Bone 2000; 26: 581-589.

20. Monti E, Mottes M, Fraschini P, Brunelli P, Forlino A,
Venturi G, et al. Current and emerging treatments for the management of osteogenesis imperfecta. Ther Clin Risk Manag 2010; 6: 367-381.

21. Astrom E, Soderhall S. Beneficial effect of long term intravenous bisphosphonate treatment of osteogenesis imperfecta. Arch Dis Child 2002; 86: 356-364.

22. Rauch F, Glorieux FH. Bisphosphonate treatment in osteogenesis imperfecta: which drug, for whom, for how long? Ann Med 2005; 37: 295-302.

23. Glorieux FH, Bishop NJ, Plotkin H, Chabot G, Lanoue G, Travers R. Cyclic administration of pamidronate in children with severe osteogenesis imperfecta. $\mathrm{N}$ Engl J Med 1998; 339: 947-952.

24. DiMeglio LA, Ford L, McClintock C, Peacock M. Intravenous pamidronate treatment of children under 36 months of age with osteogenesis imperfecta. Bone 2004; 35: 1038-1045.

25. Rauch F, Plotkin H, Travers R, Zeitlin L, Glorieux FH. Osteogenesis imperfecta types I, III, and IV: effect of pamidronate therapy on bone and mineral metabolism. J Clin Endocrinol Metab 2003; 88: 986-992.

26. Barros ER, Saraiva GL, de Oliveira TP, Lazaretti-Castro M. Safety and efficacy of a I-year treatment with zoledronic acid compared with pamidronate in children with osteogenesis imperfecta. J Pediatr Endocrinol Metab 20।2; 25: 485-49|

27. Vuorimies I, Toiviainen-Salo S, Hero M, Makitie O. Zoledronic acid treatment in children with osteogenesis imperfecta. Horm Res Paediatr 201 I; 75: 346-353.

28. Lazala $\mathrm{O}$, Solaque H. Terapia con bifosfonatos en osteogénesis imperfecta. Rev Col Or Tra 2009; 23: 109. II4.

29. Engelbert RH, Beemer FA, van der Graaf $Y$, Helders PJ. Osteogenesis imperfecta in childhood: impairment and disability--a follow-up study. Arch Phys Med Rehabil 1999; 80: 896-903.

30. Zeitlin L, Fassier F, Glorieux FH. Modern approach to children with osteogenesis imperfecta. J Pediatr Orthop B 2003; 12: 77-87.

3I. Antoniazzi F, Mottes M, Fraschini P, Brunelli PC, Tato L. Osteogenesis imperfecta: practical treatment guidelines. Paediatr Drugs 2000; 2: 465-488. 